Home $\mid$ Mailing list $\mid$ Search | Browse | Hot topics $\mid$ Matrices $\mid$ About $\mid$ Help $\mid$ Contact

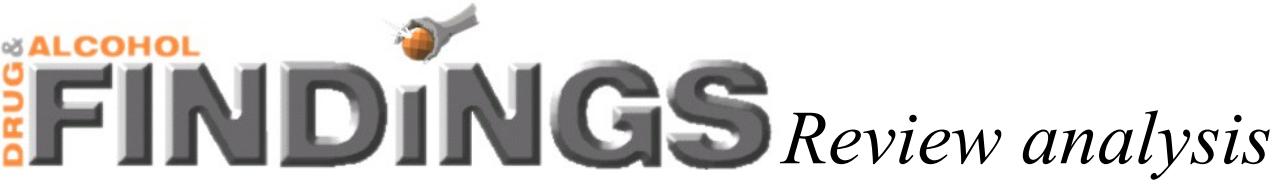

This entry is our analysis of a review or synthesis of research findings considered particularly relevant to improving outcomes from drug or alcohol interventions in the UK. The original review was not published by Findings; click Title to order a copy. Free reprints may be available from the authors - click prepared e-mail. The summary conveys the findings and views expressed in the review. Below is a commentary from Drug and Alcohol Findings.
Send email for updates

your.email@address

\section{SEND}

Links to other documents. Hover over for notes. Click to highlight passage referred to. Unfold extra text \&u乛

Copy title and link | Comment/query | Tweet

\title{
Supervised consumption sites: a nuanced assessment of the causal evidence.
}

Caulkins J.P., Pardo B., Kilmer B.

DOWNLOAD PDF

for saving to

your computer

\section{Addiction: 2019.}

Unable to obtain a copy by clicking title? Try asking the author for a reprint by adapting this prepared e-mail or by writing to Dr Kilmer at kilmer@rand.org.

How high should the evidence bar be set when deciding whether to endorse drug consumption rooms?

SUMMARY Drug consumption rooms (also known as supervised consumption sites) provide hygienic and supervised spaces for people to inject or otherwise consume illicit drugs. They operate in more than 10 countries, and have mostly emerged as 'bottom-up' responses to the harms experienced by people who inject drugs, including HIV and overdose deaths.

Efforts to summarise the evidence base ( $\left(\begin{array}{lllll}1 & 2 & 3 & 4 & 5\end{array}\right)$ have tended to reach strongly positive conclusions about drug consumption rooms. However, there is limited evidence demonstrating a 'cause and effect' relationship between drug consumption rooms and observed positive outcomes.

On the question of whether there is enough evidence to justify wider roll-out of drug consumption rooms, it is common to hear the following opposing views:

"Yes, there is sufficient evidence": One view notes that people are dying on the streets but not one person has died from an overdose in a drug consumption room. Therefore, immediate expansion is imperative and anything less would be immoral.

"No, there is insufficient evidence": Another view insists that since none of the evaluations employ 'gold standard' methods of demonstrating cause and effect, we cannot be confident in drug consumption rooms producing meaningful, positive effects.

There is, however, abundant room between those extremes, and exploring this space could help shift the response to the question from a fight over "yes" versus "no" to, "It depends on the type of decision and perspective".

Aiming to provide a more nuanced assessment of the evidence base, the featured US review 
concentrated on the findings of higher-quality research and assessed how much evidence policymakers would need to endorse drug consumption rooms.

Of the 65 articles identified, just nine (from four drug consumption rooms in three cities) compared changes in outcomes in neighbourhoods where a drug consumption room had opened to control areas where they had not - a robust research design particularly desirable in the absence of randomised controlled trials. Also reviewed were eight simulation studies that attempted to estimate the costs and benefits of existing drug consumption rooms at reducing disease transmission and overdose.

\section{Main findings}

\section{Outcomes}

The literature has reported favourable or no effects on most outcomes including overdoses. For example, one study found a significantly greater reduction in the number of fatal overdoses within 500 metres of Vancouver's Insite facility than outside that radius.

There has been no evidence that drug consumption rooms increase crime. Multiple studies ( $\left.\begin{array}{lll}1 & 2 & 3\end{array}\right)$ of the Sydney consumption room found no effect on crime and one study in Vancouver suggested that crime may have fallen after the drug consumption room opened.

Other than mixed results concerning publicly-disposed injecting equipment around a drug consumption room in Barcelona ( 12 ), no studies have reported adverse outcomes including possible unintended consequences such as prolonging drug-using careers, reducing local property values, or normalising drug use in the eyes of young people.

\section{The importance of scale}

Nobody has died from an overdose inside a drug consumption room, which serves as a strong argument for them having a positive effect. However, since drug consumption rooms only supervise a small proportion of all drug use, spillover effects on behaviour outside drug consumption rooms also matter. For context, Insite was estimated to supervise $5 \%$ of injections in its Vancouver Downtown Eastside locality in the early 2000s, leaving most drug use occurring outside the drug consumption room.

While drug consumption rooms do not have to supervise all injections in order to be effective or justifiable, it is helpful to consider what it would take to supervise all injections. Using the United States as an example, there were approximately 1.5 million people who regularly (ie, on a daily/near daily basis) used heroin circa 2016. If these people consumed heroin twice a day, that would come to about 1.1 billion episodes per year. Even if all drug consumption rooms worldwide (totalling around 100) moved to the US and expanded to be as large as Vancouver's Insite, that would still cover less than $2 \%$ of injecting episodes. Supervising all of them would require approximately 7,000 Insitelike facilities and probably cost over 10 billion US dollars per year to operate - more if use of shorter-duration opioids like fentanyl result in more injecting sessions per day ( 12 ) or if drug consumption rooms committed to supervising the use of opioid pills, cocaine, or methamphetamine. To put this into perspective, there are only about 1,500 opioid treatment providers in the US.

\section{Costs and benefits}

Costs for supervising drug use (the most distinctive function of drug consumption rooms) have been estimated at roughly the same in Vancouver and Sydney - the equivalent in Canadian currency of $C \$ 7.50-C \$ 10$ per injection. This would bring the cost of supervising all injections for someone who injects twice a day to about $C \$ 5,500-C \$ 7,300$ per year, which is in the same ballpark as the cost of providing methadone for a year to a patient in the United States. 
Focusing almost exclusively on Vancouver, simulation studies have found that the value of averting a fatal overdose or HIV infection is so high that drug consumption rooms can pass the cost-benefit test even if the number of people affected is small (1 2). However, many other interventions also pass that test, including medicationassisted treatment, needle and syringe exchanges and naloxone, raising the question of how best to distribute scarce financial resources across such interventions.

It is unclear whether greater benefit would be achieved by investing the same amount of resources in interventions other than drug consumption rooms due to a lack of evidence about the magnitude of population-level benefits - firstly, because the literature can blur the lines between the impact of a drug consumption room's entire suite of interventions and its supervision of consumption, and secondly, because supervised consumption can have spillover effects on behaviour outside drug consumption rooms as well as within the four walls.

\section{Adequacy of literature}

There is no clear-cut answer to the question of how much favourable evidence must accumulate before implementing new drug consumption rooms - the threshold may differ depending on who is making the decision:

- The politician is someone, such as a mayor, who faces a 'yes' or 'no' choice about allowing drug consumption rooms, and will be held responsible for their outcomes but not their funding or operation. The politician may care as much about the logical consistency of drug consumption rooms as having a rigorous evidence base. Furthermore, politicians may have a bias toward acting in response to a crisis, but they can be sensitive to adverse side-effects, which are amplified in today's partisan political-media discourse.

- The planner needs to understand not just whether an intervention is effective, but also how effective it is. Would implementation solve the underlying problem, make a major contribution, or be just an appreciated but minor supplement to some other primary response? And, how readily can model programmes be replicated and brought to scale?

- The pioneer isn't content with identifying the best existing intervention, but asks "How can I invent something better by teasing out the strengths and weaknesses of the existing options?" Like the planner, the pioneer cares about the magnitude of effects, but requires that information at the level of constituent interventions (eg, the supervised consumption element with drug consumption rooms that provide other services), and wants to know why, not just whether, something works.

The threshold may also differ depending on the intellectual perspective:

- The academic typically insists on having very strong evidence before concluding that a causal effect exists, but devotes less attention to interpreting the magnitude of an effect, and is less concerned about costs or cost-effectiveness.

- The advocate might bristle at perpetual calls for more (and more rigorous) research in the midst of a crisis, noting that no reasonable person would wait for a randomised control trial evaluating parachutes before donning one when leaping from a plane. From their perspective, the intervention 'makes sense' and there are more than enough reviews of the broader literature that provide nearly unanimous support for drug consumption rooms. There is also a sizeable literature representing the perspective of drug consumption room clients, not distant social planners, which investigate important but hard-to-measure outcomes, such as how drug consumption rooms reduce stigma or enhance access to social services in hard-toreach populations, rather than whether drug consumption rooms are cost-effective ways to spend taxpayers' dollars.

- The allocator of scarce resources wants to know whether drug consumption rooms save more lives per dollar than other available alternatives, such as expanding access to naloxone and opioid substitute medications.

\section{The authors" conclusions}

There is minimal evidence demonstrating a 'cause and effect' relationship between 
drug consumption rooms and favourable outcomes. However, the published literature is large and almost unanimous in its support for drug consumption rooms, and there is little to no basis for concern about drug consumption rooms producing adverse effects.

How high the evidence bar should be set when stakeholders are deciding whether to endorse drug consumption rooms depends upon the type of decision-maker and their perspective. The distinctions the featured paper drew between politicians, planners and pioneers, and between academics, advocates and allocators of scarce resources, could help different interested parties to walk these fine lines in harmony. It could also open up space to discuss how research does not currently (but could in the future) allow society to optimise the design of drug consumption rooms in order to maximise potential life-saving benefits.

FINDINGS COMMENTARY Drug consumption rooms tend to emerge from local initiatives aimed at reducing the harms of public drug consumption, and are not designed or implemented in ways that permit evaluation by the scientific 'gold standard' of a randomised controlled trial - randomly allocating people to demonstrate the causal effect of drug consumption rooms, or more specifically the supervised consumption element of drug consumption rooms. Having said that, there are examples of robust research designs in the evidence base, including nine studies identified in the featured paper which compared changes in outcomes in neighbourhoods where a drug consumption room had opened versus control areas where they had not.

In 2006, the UK Independent Working Group on Drug Consumption Rooms concluded that "the methodological problems involved ... should not detract from [drug consumption rooms'] considerable success". On balance, the evidence suggests that drug consumption rooms can have a positive impact on the health of their clients, for example through ensuring (relatively) safe and hygienic injecting in the facility, providing personalised advice and information on safe injecting practices, recognising and responding to emergencies, and providing access to a range of other on-site and off-site interventions and support. And, even without a randomised trial, it may be possible to at least estimate the likelihood that an intervention (in this case, a drug consumption room) is having a positive or negative impact. For instance, it may not be possible to determine impacts on the transmission of infectious diseases, but it is possible to observe impacts on self-reported needle and syringe sharing - the main cause of transmission among people who use drugs.

The featured paper made a unique contribution to the debate about drug consumption rooms, diverging from the well-trodden narrative that the evidence base provides almost unanimous support for safer injecting facilities, to look at gaps in the evidence base and the adequacy of the research depending on who is reading it. The distinctions made between different stakeholder groups may be useful in helping to establish a more nuanced attitude to drug consumption rooms by looking at the interests of those most likely to be involved in discussions and decisions around them being established. This could serve to facilitate the provision of drug consumption rooms by anticipating the variety of views and possible opposition. However, the authors may have been over-optimistic if their goal for the paper was to facilitate "harmony" between stakeholders with divergent perspectives.

"We hope that the distinctions we have drawn, between decisionmakers who are politicians, planners and pioneers, and between the perspectives of academics, advocates and allocators of scarce resources, help different interested parties to walk these fine lines in harmony." 
The analysis and commentary elucidated the reasons for different appraisals of the evidence base according to decision-making and intellectual responsibilities, but did not address the ideological struggle between acceptance and zero-tolerance of illegal drug use at the root of the debate, the power dynamics between stakeholders (eg, the way that politicians may act as or be perceived as gatekeepers or roadblocks to lifesaving interventions), or the overlap between stakeholder groups (eg, people whose academic area of study is motivated by social justice).

There are currently no drug consumption rooms in the UK, despite concerns over the harm caused by public injecting, and concerns that the needs of the target group of drug consumption rooms are continuing not to be met by existing services alone. According to recent statements from the Home Office and Prime Minister, the UK Government has "no intention" (1 2) and "no plans" to introduce drug consumption rooms.

Other interventions identified in the paper may serve some of the functions of drug consumption rooms, however, may not all be equally accessible to the target group of drug consumption rooms. For example, some would seem to be appointment-based rather than, as with drug consumption rooms, attended on a drop-in basis. Therefore, when the authors posed the (understandable) question about whether greater benefit would be achieved by investing the same amount of resources in interventions other than drug consumption rooms, they excluded the more fundamental argument about why drug consumption rooms should be considered in addition to existing interventions.

Thanks for their comments on this entry in draft to Blaine Stothard, Co-Editor of Drugs and Alcohol Today. Commentators bear no responsibility for the text including the interpretations and any remaining errors.

Last revised 24 September 2019. First uploaded 28 August 2019

- Comment/query

- Give us your feedback on the site (two-minute survey)

- Open Effectiveness Bank home page

Add your name to the mailing list to be alerted to new studies and other site updates

Top 10 most closely related documents on this site. For more try a subject or free text search

MATRIX CELL 2017 Drug Treatment Matrix cell E1: Local and national systems; Reducing harm

HOT TOPIC 2016 Time for safer injecting spaces in Britain?

STUDY 2014 Drugs: international comparators

REVIEW 2017 An evidence review of the outcomes that can be expected of drug misuse treatment in England

REVIEW 2015 Drug consumption rooms: an overview of provision and evidence

REVIEW 2014 A review of buprenorphine diversion and misuse: the current evidence base and experiences from around the world

DOCUMENT 2014 Needle and syringe programmes

MATRIX CELL 2017 Drug Treatment Matrix cell D1: Organisational functioning; Reducing harm

MATRIX CELL 2017 Drug Matrix cell A1: Interventions; Reducing harm

STUDY 2010 Were the changes to Sweden's maintenance treatment policy 2000-06 related to changes in opiate-related mortality and morbidity? 